\title{
A framework for participatory decision support using Pareto frontier visualization, goal identification and arbitration
}

\author{
Roman Efremov ${ }^{a, 1}$, David Rios Insua ${ }^{a}$, Alexander Lotov $^{b}$ \\ ${ }^{a}$ Statistics and Decision Sciences Group, Rey Juan Carlos University, Tulipan s/n, 28933, Madrid, Spain \\ ${ }^{b}$ Department of Economic Decision Analysis Dorodnicyn Computing Centre of the Russian Academy of Sciences, Vavilov \\ st. 40, 119333 Moscow, Russia
}

\begin{abstract}
There is a growing interest in promoting participation of lay stakeholders in public decision making processes, possibly with the aid of Internet based systems. This implies supporting non-sophisticated users and, consequently, developing userfriendly, yet rigorous, participatory decision support methods. We outline a framework to develop such methods based on interactive Pareto frontier visualization combined with expression of preferences in terms of feasible goals and using feasible goal-based arbitration.

Keywords: Decision analysis, Participatory Decision support, Pareto frontier visualization, Feasible Goals, Arbitration methods, EDEMOCRACY
\end{abstract}

\section{Introduction}

Possibly because of the almost ubiquitous presence of Internet, people are gradually asking for further involvement in public policy decision making. The last few years have brought numerous attempts to formalize and implement different approaches to facilitate this process. As an example of active penetration of public opinion in political decision making, we should mention participatory budgets, see e.g. Ríos et al. (2007). They constitute an attempt to allow the general public to have a word and aid in deciding and approving how public budgets, mainly in municipalities, are spent. However, no formal negotiation or participatory decision support tools are usually employed, there being little methodology available in this field. This problem is becoming more acute as more complicated issues are being faced by the general public. As an example, the concept of public participation is transforming policies, as may be seen, e.g., in the European environmental directives which embody and promote public participation as an integral element, see e.g. the Water Framework Directive (European Commission Directive/2000/60/EC).

Information Technologies (IT) provide new potential to support dispersed stakeholders, both geographically and socially. Indeed, many authors have dwelt on how Internet is changing the way people interact with governments, see e.g. Browning (2002). However, so far, most ideas relating Internet and politics have been directed towards facilitating traditional political methods through IT as with electronic voting instead of voting with a piece of paper. The most challenging goal, however, is to transform, rather than facilitate, public decision-making processes, through the use of IT.

\footnotetext{
${ }^{1}$ Corresponding author. Tel.: +34 91488 8132; fax: +34 914887626 E-mail address: roman.efremov@urjc.es
} 
There are already several examples of systems used or about to be used for public decision-making support, see, for example, Ríos and Ríos Insua (2007), Castelletti and Soncini-Sessa (2006) and Dietrich et al. (2006). Also, there are attempts of standardization of the wide diversity of tools available, their inventory and their assembly under a common basis, see French et al. (2007).

A participatory decision making process could be divided into two stages, once the problem has been formulated. In the first stage, stakeholders express their preferences in some form. In the second stage, such preference information is used to construct the participatory decision. It is clear that the information should be provided to and extracted from the stakeholders in a meaningful way at the first stage of participatory decision processes. Therefore, emphasis should be placed on simplicity and user-friendliness. This does not entail, however, neglecting rigorousness of the implemented methods.

It is important to remark that a description of the stakeholders' interests will be usually based on several performance indicators, an important feature of public decisionmaking problems. As an example, we could demand that a municipal budget should satisfy various needs of the local population like providing more safety, mitigate environment impact or increase employability. Knowing these needs facilitates the development of several decision criteria that must be considered in the decision making problem. Some key references in multicriteria optimization theory and methods include Keeney and Raiffa (1976), Steuer (1986) or Miettinen (1999).

Given the above prerequisites, we describe a framework for participatory decision support. Our aim is to develop a user-friendly procedure potentially implementable on the web that can collect information about the stakeholders' preferences and transform this information into a decision in a fair and transparent manner. The procedure must be simple enough for lay stakeholders who, in general, will not be quantitatively sophisticated. In particular, the preference information must be obtained from stakeholders in an understandable way. On the other hand, such information must be sufficient to be used in a rigorous arbitration scheme.

We propose here to restrict the preference information provided by the stakeholders to their goals. Thanks to their simplicity, goal methods have found broad applications, see e.g. Charnes and Cooper (1961), Ignizio (1985) or Romero (1991). However, goal based methods have a disadvantage: if the feasibility information is unknown, the goals may turn out to be too ambitious or too pessimistic. In the case of experts, this problem may be not too important, since experts usually understand feasibility frontiers and are able to locate their goals reasonably. However, it is virtually impossible to hope that lay stakeholders will have such capacity.

To solve the problem, we suggest informing the stakeholders about the feasibility frontier, which, in the framework of multicriteria optimization problems is the same as the Pareto (non-dominated) frontier. This information should aid stakeholders in applying goal-based methods comprehensively. Multicriteria optimization theory has provided several ideas on how to inform decision makers about the Pareto frontier, see e.g. Cohon (1978), Steuer (1986) or Miettinen (1999). Most of them are based on the presentation of a list of feasible points in the criterion space, which approximate the Pareto frontier. However, studying such large lists is usually cumbersome, even for experts, see Larichev (1992). Clearly, it would be too complicated for lay stakeholders.

We simplify the process of informing the stakeholders about the Pareto frontier through its visualization. When there are more than two criteria, visualization of the Pareto frontier may be carried out through the Interactive Decision Maps (IDM) tech- 
nique, see Lotov et al. (2004a). The point of the Pareto frontier preferred by the decision maker is often denoted as the feasible goal. Its identification, which is supported through Pareto frontier visualization and leads to the associated decision, is known as the Feasible Goals Method (FGM). The combination of FGM with IDM has been implemented in several software systems which are simple graphical tools for decision support that inform users about the Pareto frontier on multicriteria decision problems and lead them to the preferred decisions through goal identification. Some applications of the FGM/IDM technique to support lay stakeholders on Internet are discussed in Lotov (2003). ${ }^{2}$

In our framework, we use the IDM technique to support stakeholders in identifying their feasible goals. Since stakeholders supported by visualizing the Pareto frontier are able to identify their feasible goals, we associate these with the maxima of the value functions of stakeholders over the Pareto frontier. This interpretation of goals will be used at the second stage of our procedure to construct a decision rule, through several goal-based arbitration methods.

The outline of the paper is as follows. In Section 2, we describe general features of our approach. Section 3 describes the IDM technique and its application to goal identification. In Section 4, we describe arbitration schemes that can be applied with the identified feasible goals. Section 5 describes some experimental results. We end up with some discussion.

\section{Description of the approach}

In this section, we describe the key features of our approach. Remember that we divide the decision process in two stages. First, the stakeholders express their preferences; then arbitration is used.

Let us introduce some notation. Let $X$ be the feasible decision set and $f: X \rightarrow \mathbf{R}^{d}$ be a mapping from $X$ to the criterion space $\mathbf{R}^{d}$ : the performance of each feasible decision $x \in X$ is described by the criterion vector $y=f(x)$, with $d$ criteria $y_{i} . Y:=f(X)$ is called the feasible criterion set. We shall assume that $X$ is compact and $f: X \rightarrow \mathbf{R}^{d}$ is continuous.

Without loss of generality, we shall assume that the criteria must be maximized. We shall say that $y$ dominates $y^{\prime}$ (in a Pareto sense) if, and only if, $y \geq y^{\prime}$ and $y \neq y^{\prime}$. The Pareto frontier of $Y$ is defined as

$$
P(Y):=\left\{y \in Y:\left\{y^{\prime} \in Y: y^{\prime} \geq y, y^{\prime} \neq y\right\}=\emptyset\right\} .
$$

Let $\mathbf{R}_{-}^{d}$ be the non-positive orthant in $\mathbf{R}^{d}$. The set $H(Y)=Y+\mathbf{R}_{-}^{d}$ is known as the Edgeworth-Pareto Hull of $Y$. $H(Y)$ is the maximal set satisfying $P(H(Y))=P(Y)$. Let us denote the criterion point $B(Y)$, defined by $B_{j}(Y):=\max _{y \in Y} y_{j}, j=1, \ldots, d$, as the bliss point. Since $Y$ is compact, the bliss point exists.

We shall assume that the $k$-th stakeholder preferences over criterion vectors $y$ can be described by a value function $v_{k}(y)$, where $k=1, \ldots, K$. However, we shall not assume that we explicitly know the stakeholders' value functions. Though constructing the value function can be used to support negotiations over the web, see e.g. Kersten et al. (2004), such methods may be too complicated for lay stakeholders, because of the

\footnotetext{
${ }^{2}$ The interested reader can find information about the FGM/IDM technique and download demo software from www.ccas.ru/mmes/mmeda/soft. There is also a web implementation of the IDM technique for finite choice, available at www.ccas.ru/mmes/mmeda/rgdb/index.htm.
} 
large volume of preference information that must be provided. For this reason we propose an alternative method for eliciting preference information: stakeholders have to identify their feasible goals, that is, their preferred feasible criterion points $y^{k} \in Y, k=1, . ., K$. If the assumption of the existence of the value function holds, the individual preferences of the $k$-th stakeholder will lead to the individually preferred decision $x^{k} \in X$ that solves the optimization problem

$$
\max v_{k}(f(x)) \text { s.t. } x \in X,
$$

so that $y^{k}=f\left(x^{k}\right)$. If the stakeholder's value function $v_{k}(y)$ is increasing with respect to Pareto dominance, that is, $v_{k}(y)>v_{k}\left(y^{\prime}\right)$ if $y \geq y^{\prime}, y \neq y^{\prime}$, the solutions of the corresponding optimization problem (1) will be non-dominated, that is, $y^{k} \in P(Y), k=$ $1, . ., K$, see Podinovski and Nogin (1982).

In our case, since the value functions $v_{k}(y)$ are not known, we cannot help the stakeholders by solving their optimization problems (1). For this reason, the $k$-th stakeholder will have to identify his preferred non-dominated point $y^{k} \in P(Y)$ by himself. However, in this process, the stakeholder is supported by the IDM technique, which informs him about the Pareto frontier as described in the next section. Identification of the feasible goals $\left\{y^{k}\right\}_{k=1, \ldots, K}$, as described above, forms the first stage of the procedure.

At the second stage, an arbitration method could be applied to select the participatory decision on the basis of the feasible goals $\left\{y^{k}\right\}_{k=1, \ldots . K}$. A review of bargaining theory, which provides the mathematical basis for arbitration methods is, for example, presented in Thomson (1994). Bargaining theory focuses on outlining desirable properties of the bargaining outcome and on how such outcome may be computed. Although various solutions suggested in the theory can provide a model for arbitration processes, sometimes it is difficult to apply them in practice, since this application usually assumes that value functions of participants are provided. As argued above, this may be complicated in the case of lay stakeholders. Instead, we adapt arbitration methods to deal with smaller volumes of preference information.

We provide now a description of the IDM technique and, then, discuss several possible arbitration schemes.

\section{The IDM technique}

\subsection{Introduction to IDM}

The IDM technique, see Lotov et al. (2004a) for details, develops an idea introduced by Gass and Saaty (1955) for bi-criteria linear problems, showing that the visualization of the Pareto frontier is possible and practical at least in that type of problems. The IDM technique displays the Pareto frontier, for more than two criteria, through interactive display of bi-criterion slices of the set $H(Y)$.

A bi-criterion slice is defined as follows. Let $\left(y_{1}, y_{2}\right)$ designate two criteria, the socalled "axis" criteria, and $z$ denote the remaining criteria, which we shall fix at $z^{*}$. A bi-criterion slice of $H(Y)$, parallel to the criterion plane $\left(y_{1}, y_{2}\right)$ and related to $z^{*}$ is defined as

$$
G\left(H(Y), z^{*}\right)=\left\{\left(y_{1}, y_{2}\right):\left(y_{1}, y_{2}, z^{*}\right) \in H(Y)\right\} .
$$

Note that a slice of $H(Y)$ contains all feasible combinations of values for the specified criteria when the values of the remaining criteria are not worse than $z^{*}$. 
Bi-criterion slices of $H(Y)$ are used in the IDM technique to display decision maps. To define a particular decision map, the user has to specify a "third", or colour-associated, criterion. Then, a decision map is a collection of superimposed slices, for which the values of the colour-associated criterion change, while the values of the remaining criteria are fixed. If one compares the slices of $H(Y)$ for two different values of the colour-associated criterion, the slice for the worst value of this criterion encloses the slice for its better value. For this reason, frontiers of slices (trade-off curves) provided at a decision map do not intersect. An example of a decision map for a convex $H(Y)$ is provided in Figure 3.1 coming from an agricultural planning problem, see Lotov et al. (2004a). A lake is used

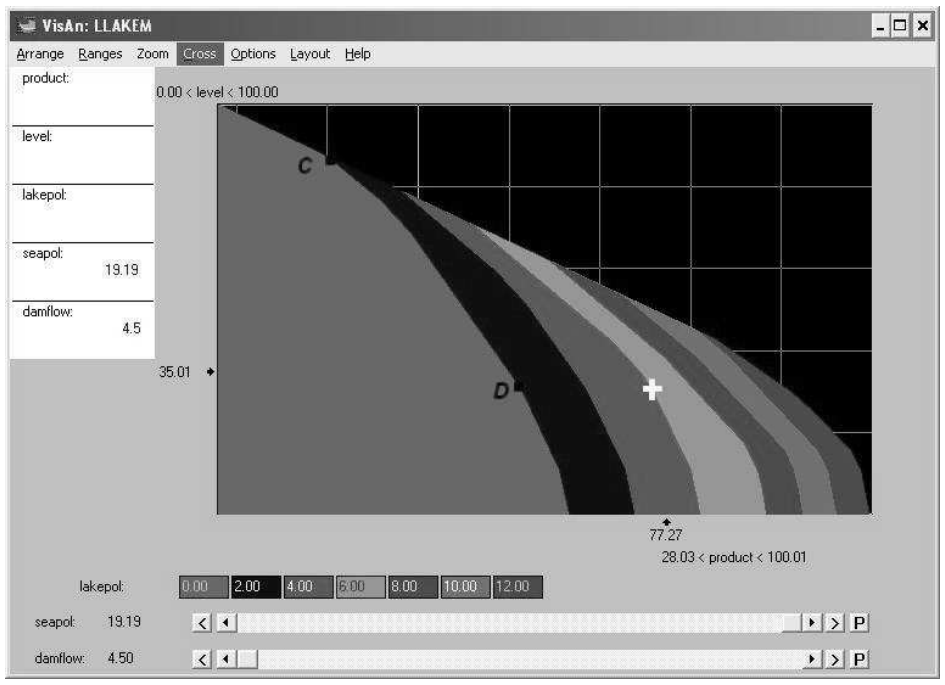

Figure 1: A decision map (gray scale variant).

for irrigation purposes. It is also a recreational zone for nearby residents. The conflict is described by three criteria: agricultural production, lake level, and lake pollution. In Figure 3.1, the trade-off curves, production $\left(y_{1}\right)$ versus lake level $\left(y_{2}\right)$, are depicted for several pollution $(z)$ values. The constraints imposed on pollution are specified by the gray scale located under the decision map. Any trade-off curve defines the limits of what can be achieved. The internal trade-off curve, marked by points $C$ and $D$, is related to minimal, i.e. zero, pollution. The trade-off curve shows how the lake level is exchanged for production, while pollution level remains at zero. Note that it is necessary to exchange a substantial drop in the level (about 30\% starting at point $D$ ) for a small increase in production needed to achieve its maximal feasible value.

Note that as the pollution level increases, the production level increases as well. Nevertheless, if the lake level is reasonably high, the trade-off curves are close to each other, which means that, for these lake levels, even a substantial increment in pollution does not result in economic advantages.

Let us discuss how the user may choose his goal on a Pareto frontier with the help of IDM. It is known that a person is reasonably capable of identifying the goal at a trade-off curve, e.g. at the curve $C D$ in Figure 3.1, see Roy (1972). The user can choose a trade-off curve by fixing the value of the (third) criterion represented with a coloured scale. He would mentally change trade-off curves improving the value of the third criterion until he finds that the values of both axis criteria become unjustifiably bad and, vice versa, would improve values of the two axis criteria until the value of the third criterion is unjustifiably 
worsened. Once having found the appropriate trade-off curve, the user would proceed by finding a compromise between the values of both criteria on the axis.

If there are more than three criteria, the user may specify the values of a fourth, fifth and more criteria by applying scroll-bars, see Figure 3.1, that help to specify these values manually. By moving scroll-bar sliders, the user can study how variations of these values influence a decision map. The animation of decision maps, which is a display of decision maps related to the automated changing value of, say, the fourth criterion, is also possible. The rates of expansion, or contraction, of coloured slices on a decision map inform the user about how the values and tradeoffs between the three criteria displayed by the decision map change while a fourth criterion changes. As the fourth criterion is represented with a scroll-bar, its deviation from its best value is also easily perceived. Once having found the compromise between decision maps and values of the fourth and fifth criteria, the user goes on with the analysis of a decision map.

In our study we restrict attention to convex $H(Y)$. Though decision maps for nonconvex $H(Y)$ have been developed, they look too complicated for lay stakeholders, see Lotov et al. (2004a), who describes a possible way out by considering the convex hull of a non-convex $H(Y)$, and then analyse this set.

\subsection{Intelligibility of the IDM technique for lay stakeholders}

As it has been mentioned, a key issue in tools supporting participatory decision making is the way in which information is provided to stakeholders. To make information intelligible, user-friendliness and representation simplicity are required. Hence, the most important question related with the application of the IDM technique is whether it is effective, i.e. whether people are able to understand decision maps.

Information visualization is usually a very efficient approach to convey information to stakeholders. McQuaid et al. (1999) suggest three key requirements to be met in information visualization: simplicity, meaning easily understandable visualization; persistence, meaning propensity to linger in the mind of the beholder; and, completeness, meaning depiction of all relevant information in data.

In the case of IDM, these requirements seem to hold true due to the parallelism between decision and topographic maps: as trade-off curves do not intersect in decision maps, they look like contour lines in a topographic map. Indeed, a value of the colourassociated criterion, which is related to a tradeoff curve, plays the role of ground elevation related with a contour line in a topographic map. One can recognize the combinations of the "axis" criteria that are feasible for a given restriction imposed on the value of the colour-associated criterion (as "places higher than..." or "places lower than..."). Moreover, one can easily understand which values of the colour-associated criterion are feasible for a given combination of the "axis" criteria (as "elevation of this place is between..."). If the distance between trade-off curves is small, this means that a small move of the trade-off curve requires a substantial change of the value of the colour-associated criterion.

Based on experiments with university students, see Lotov et al. (1998), as well as on our experiments outlined in Section 5, we conclude that the IDM technique is, indeed, simple enough, at least for people with university background. 


\subsection{Implementation aspects of the IDM technique}

In the framework of IDM, $H(Y)$ is approximated in advance. Effective stable methods for approximating a convex $H(Y)$ were proposed in Bushenkov and Lotov (1982), and implemented in the IDM software, see Lotov et al. (2004a) for details.

Note that the approximation of $H(Y)$, which requires up to $99 \%$ of the computing efforts, can be separated from the human-computer exploration of decision maps and can be performed automatically. Moreover, slices of an approximation of $H(Y)$ can be computed instantaneously. This feature of the IDM technique facilitates implementation on computer networks and decision maps to be depicted and animated on-line. The technique is applied on the Internet through a web client-server architecture: approximating the set $H(Y)$ is accomplished on a server, while exploration of the Pareto frontier is carried out by means of Java applets on the user's computer, as explained in Lotov et al. (2004a, b). A scheme of an implementation of the IDM technique in the web is provided in Figure 2. A Java applet that can display decision maps in an interactive mode is transmitted to the user's computer. The applet can be used to identify the feasible goal, too. After fixing the goal, it is transmitted to the web-server. If FGM is used, the web-server computes the related decision alternative and provides it to the user, which receives the goal-related decision almost immediately.

Lotov et al. (1999, 2001, 2004a) and Lotov (2003) provide applications of the IDM

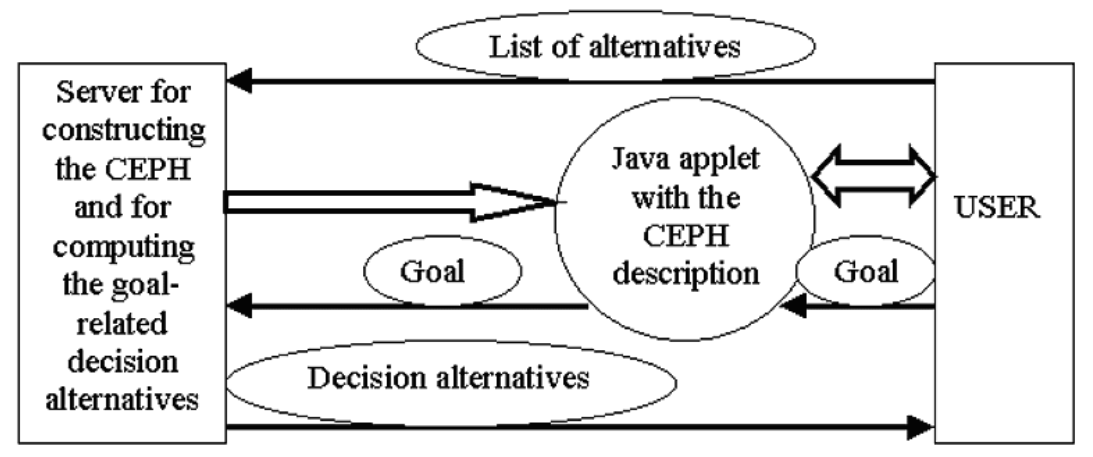

Figure 2: Scheme of the Internet resource.

technique in public decision making problems. Dietrich et al. (2006) describe a real-life application of the IDM technique on the web.

\section{Arbitration methods based on feasible goals}

\subsection{Approaches to arbitration}

The IDM technique can be used also for negotiation support, as proposed in Lotov et al. $(1998,1999)$. These schemes implement the concept of Principled Negotiations, see Raiffa (1982), that is, negotiations that focus on interests, rather than on particular positions. To find a compromise solution in such negotiations, negotiators must develop some concessions that may not directly appear in the model.

We propose here another way of using the IDM technique for participatory decision support. Unlike to the previous endeavours, we concentrate on finding a satisfactory 
arbitration scheme that automatically computes the decision, once the stakeholders' preferences are specified. We shall describe two possible types of arbitration schemes. The first type is based on heuristics. In the second type, the feasible goal of the $k$-th participant is used to construct his surrogate value function, as we exemplify in Appendices 1 and 2 .

\subsection{Heuristic arbitration methods}

Various heuristic arbitration schemes can be proposed based on the notion of feasible goals. In this section we consider one class of heuristic rules that is sufficiently simple to be understood by lay stakeholders, but has relevant properties. It is based on calculating the gravity centre of the feasible goals and on subsequently maximizing a scalar objective function.

The class of arbitration rules looks as follows. First, the gravity centre of the feasible goals $\left\{y^{k}\right\}_{k=1, \ldots, K}$ is calculated, i.e.

$$
y^{g}=\frac{1}{K} \sum_{k=1}^{K} y^{k} .
$$

Since $H(Y)$ is convex, $y^{g} \in H(Y)$. However, it will not usually be Pareto-optimal. Thus, we have to refine the point $y^{g}$ to obtain a Pareto optimal point. To do it, we can use various scalar objective functions $\varphi\left(y, y^{g}\right)$, which evaluate the gains by refining the gravity centre $y^{g}$. The arbitration decision $x^{a}$ can be found by solving the following optimization problem

$$
\max _{x \in X} \varphi\left(f(x), y^{g}\right) .
$$

The arbitration point in the criterion space is given by $y^{a}=f\left(x^{a}\right)$.

The simplest scalar objective function is the linear one:

$$
\varphi_{l}\left(y, y^{g}\right):=\sum_{i=1}^{d} w_{i}\left(y_{i}-y_{i}^{g}\right) .
$$

where $w=\left(w_{1}, w_{2}, \ldots, w_{d}\right)$ is a vector of positive weights. The linear scalar objective function is very sensitive to the shape of $H(Y)$ and to the weights, which must be given in advance. Thus, the application of the linear scalar objective function may result in an arbitration point $y^{a}$, which has totally different proportions of the criteria values as compared with the proportions of the gravity centre, see Appendix.

Another function that improves upon the gravity point is the scalar objective function proposed by Germeier (1970):

$$
\varphi_{g}\left(y, y^{g}\right):=\min _{i=1, \ldots, d} \lambda_{i}\left(y_{i}-y_{i}^{g}\right)
$$

where $\lambda=\left(\lambda_{1}, \ldots, \lambda_{d}\right)$ is a vector of positive weights, say, $\lambda_{i}=1$ for all $i=1, \ldots, d$. This function refines the gravity point in the sense of weak Pareto dominance, see Figure 3 where the Pareto frontier is given by the bold line and level contours of Germeier function are given by ordinary lines, whereas the feasible goals of two stakeholders $y^{1}$ and $y^{2}$, the gravity centre $y^{g}$ and the arbitration point $y^{a}$ are displayed by circles. Thus, the proportions of the criterion values in the arbitration point cannot differ too much from these proportions in the gravity centre. 

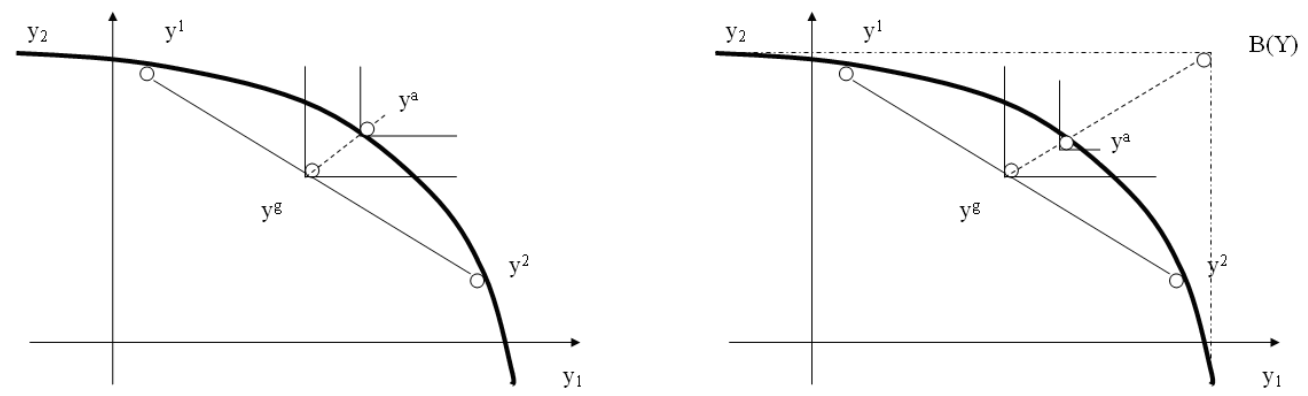

Figure 3: Improving the gravity centre with Germeier function (left) and Chebyshev function (right)

Evidently, the arbitration point dominates the gravity centre. A minor disadvantage of Germeier's function is that the solution of the corresponding problem (3) is a weak Pareto optimal decision. However, this disadvantage can be easily removed by adding a linear expression $\varepsilon \sum_{i=1}^{d}\left(y_{i}-y_{i}^{g}\right)$ with a small positive parameter $\varepsilon$. The modified Germeier function results in a Pareto optimal decision.

Another possible scalarizing function, which improves upon the gravity point, is the Chebyshev distance from the bliss point, discussed in detail in Steuer (1986)

$$
\varphi_{C h}(y, B(Y), \lambda):=\max _{i=1, \ldots, d}\left\{\lambda_{i}\left(B_{i}(Y)-y_{i}\right)\right\} .
$$

This function evaluates the deviation of $y \leq B(Y)$ from the bliss point $B(Y)$. To refine the gravity center $y^{g}$, one simply needs to minimize the Chebyshev distance from the bliss point for the weights, which are inverse to the coordinate-wise differences between the bliss point and the gravity centre, i.e.

$$
\lambda_{i}=\frac{1}{B_{i}(Y)-y_{i}^{g}},
$$

where $y_{i}^{g}$ is not equal to $B_{i}(Y)$ for $i=1, \ldots, d$. The level lines of this function are given in Figure 3. One can see that improving the value of the Chebyshev function with such parameter values results in refining the gravity centre in the sense of Pareto dominance. Thus, the proportions of the criterion values in the arbitration point and in the gravity centre cannot differ too much from each other, as in the case of Germeier's function.

Another class of functions, which can improve the gravity point for constructing an arbitration solution, is provided by Wierzbicki's achievement scalarizing functions, see Wierzbicki, (1982). Its simplest form is close to Germeier's function:

$$
\varphi_{W}\left(y, y^{g}\right):=\min _{i=1, \ldots, d} w_{i}\left(y_{i}-y_{i}^{g}\right)+\rho \sum_{i=1}^{d} w_{i}\left(y_{i}-y_{i}^{g}\right),
$$

where $\rho$ is a positive value. If $\rho$ is small, it coincides with the modified Germeier function. Otherwise, Wierzbicki's function acquires some properties that help to describe possible substitution between criterion values.

Let us consider now some of the properties of the arbitration decision $x^{a}$ and arbitration criterion point $y^{a}$ obtained resolving (3). As we have already said, for any reasonable scalar function $\varphi\left(y, y^{g}\right)$ which is increasing with respect to Pareto dominance, that is, $y \geq y^{\prime}, y \neq y^{\prime}$ results in $\varphi\left(y, y^{g}\right)>\varphi\left(y^{\prime}, y^{g}\right)$, the solution of (3) is Pareto optimal, see Podinovski and Nogin (1982). It is clear that the solution has the symmetry 
and anonymity properties (Thomson, 1994) because the gravity centre possesses such properties.

In addition, the following important property holds: the solution is independent of irrelevant alternatives. Namely, if $X^{\prime} \subset X$ such as $x^{k} \in X^{\prime}$ for $k=1, \ldots, K x^{a} \in X^{\prime}$ and $B(f(X))=B\left(f\left(X^{\prime}\right)\right)$, then $x^{a}$ is the solution of the arbitration procedure for the problem, in which the feasible decision set $X^{\prime}$ is used instead of the set $X$. To prove it, it is sufficient to note that the solution $x^{a}$ of $(3)$ does not change if the set $X^{\prime}$ is used instead of the set $X$.

\subsection{Arbitration rules based on surrogate value functions}

Another approach to construct arbitration schemes can be based on using the feasible goals to approximate value functions. Such approach provides an opportunity to apply the results from bargaining theory, see Thomson (1994).

Let us first describe the key concepts. We shall use the feasible goal $y^{k}$ provided by the $k$-th stakeholder to identify the surrogate value function $\tilde{v}^{k}(y)$ used to model his preferences. The surrogate value function of the $k$-th stakeholder $\tilde{v}^{k}(y)$ must be selected from a class of parametric value functions $\tilde{v}(y, \alpha)$ where $\alpha$ is a parameter vector that belongs to some set. We use the word "surrogate" to stress that the information about a goal is not sufficient to identify the actual value function. Thus, we require that the class of parametric value functions $\tilde{v}(y, \alpha)$ is such that one can identify the parameter values by using a single Pareto-optimal criterion point, namely, the feasible goal $y^{k}$ provided by the stakeholders.

After identifying the surrogate value functions of all stakeholders, one can apply a wide class of arbitration schemes, see e.g. Thomson (1994). Note, that this freedom for selecting the surrogate value functions and arbitration schemes may, sometimes, result in some undesirable properties of the arbitration points. We provide an example in Appendix.

\subsubsection{Constructing the surrogate value functions}

In this section, we have to restrict ourselves to convex $H(Y)$ such that for any $y \in$ $P(H(Y))$ the normal vector of $P(H(Y))$ at $y$ is positive. This is satisfied, e.g., by polyhedral sets. Note that in the framework of IDM, $H(Y)$ is approximated as a polyhedral set given by a system of linear inequalities.

Let us consider several examples of parameter identification for the surrogate value functions based on feasible goals. The simplest example of a class of surrogate value functions is given by the linear functions

$$
\tilde{v}(y, w):=\sum_{i=1}^{d} w_{i} y_{i},
$$

where $w=\left(w_{1}, \ldots, w_{d}\right)$ is a vector of positive weights which plays the role of parameters for the linear value function. Since $H(Y)$ is linear and we use linear surrogate value functions, the feasible goal of the $k$-th stakeholder $y^{k}$ must be a solution of the linear programming problem

$$
\max _{y \in H(Y)} \tilde{v}^{k}(y, w)
$$

According to the Karush-Kuhn-Tucker (KKT) optimality condition, which is necessary and sufficient in the linear case, $w$ must belong to the cone of normal vectors of the 
supporting hyperplane for $H(Y)$ at the solution of (5). In the simplest case of a single supporting hyperplane at $y^{k}$ (a single active linear constraint of $H(Y)$ in $y^{k}$ ), it is sufficient to select its normal vector $c^{k}$ for $w$ to obtain $y^{k} \in P(Y)$ as one of the solutions of (5). When there are several active constraints (a cone of normal vectors to supporting hyperplanes of $H(Y)$ exists at $\left.y^{k}\right)$, one can use one of these vectors, say, the average of the wedges of the cone.

Now, let us consider the surrogate value functions in the class of concave continuously differentiable value functions. Again, we will identify parameters of such surrogate value functions with the aid of the KKT condition: the gradient of the value function at the optimal point $y^{k}$ must belong to the cone of normal vectors to supporting hyperplanes. Let us assume, for simplicity, that $H(Y)$ has a single supporting hyperplane at $y^{k}$. Let $c^{k}$ be its normal vector. Then, the KKT optimality condition says that the vector $c^{k}$ must be collinear with the gradient of the value function $\tilde{v}^{k}(y)$ of the $k$-th stakeholder at the point $y^{k} \in P(Y)$, i.e.

$$
c^{k}=b^{k}\left(\frac{\partial \tilde{v}^{k}}{\partial y_{1}}, \ldots, \frac{\partial \tilde{v}^{k}}{\partial y_{d}}\right),
$$

with $b^{k}$ a positive constant. $c^{k}$ is a positive vector since $y^{k} \in P(Y)$ and $H(Y)$ is polyhedral. Often this condition is sufficient to identify the parameters of the value function. by

As an example, let us consider the value function $\tilde{v}(y, \alpha)$ defined for $y_{i}>0, i=1, \ldots, d$

$$
\tilde{v}(y, \alpha):=\prod_{i=1}^{d} y_{i}^{\alpha_{i}}, 0<\alpha_{i}<1
$$

The gradient of (7) at $y^{k}$ is

$$
\left(\frac{\alpha_{i} \tilde{v}\left(y^{k}, \alpha\right)}{y_{i}^{k}}, i=1, \ldots, d\right) .
$$

Thus, the vector $c^{k}$ and the gradient of the value function are collinear if $c_{i}^{k}=b^{k} \frac{\alpha_{i}}{y_{i}^{k}}, i=$ $1, \ldots, d$. Thus, one can take $\alpha_{i}=c_{i}^{k} y_{i}^{k}, i=1, \ldots, d$. If there exists a cone of vectors of supporting hyperplanes at the feasible goal $y^{k}$, one can use the average vector of the cone to identify the parameters of the function.

Specification of parameters for the Chebyshev function $\varphi_{C h}(y, B(Y), \lambda)$ introduced in (4) is described in the next subsection, where an example of an arbitration rule based on a surrogate value function is considered.

\subsubsection{Arbitration based on averaging parameters of Chebyshev regret func- tions}

The goal-based arbitration rule applies the weighted Chebyshev function (distance) (4). The individual goals of participants are considered as feasible criteria points, which provide the minima of individual Chebyshev distances from the bliss point. These distances are interpreted as the regrets of participants related to the evidence that they cannot obtain maximal values of all criteria simultaneously.

For a given parameter vector $\lambda$, the feasible point $s(B(Y), \lambda)$ closest to $B(Y)$ in the sense of the Chebyshev function $\varphi_{C h}(y, B(Y), \lambda)$, can be found by solving

$$
\min _{y \in H(Y)} \varphi_{C h}(y, B(Y), \lambda) \text {. }
$$


Since $Y$ is compact, the solution $s(Y, \lambda)$ of (8) exists for any $\lambda \in \mathbf{R}_{+}^{d}$. It is easy to see that the Chebyshev function is non-decreasing with respect to Pareto dominance, that is,

$$
\varphi_{C h}(y, B(Y), \lambda) \geq \varphi_{C h}\left(y^{\prime}, B(Y), \lambda\right) \text { if } y \geq y^{\prime}, y \neq y^{\prime} .
$$

Since $H(Y)$ is convex, one can prove that $s(Y, \lambda)$ is unique and $s(Y, \lambda) \in P(Y)$.

Let us consider now the inverse problem: how to find parameters $\lambda^{k}$ on the basis of the given feasible goal point $y^{k}, k \in\{1, \ldots, K\}$. To do it, one needs to assure that $y^{k}<B(Y)$. As usual, see Steuer (1986), we guarantee this by considering a modification of the bliss point

$$
B_{i}^{*}(Y):=B_{i}(Y)+\epsilon_{i}, i=1, \ldots, d,
$$

where $\epsilon_{i}$ are small positive numbers. Let

$$
\lambda_{i}^{0 k}:=\frac{1}{B_{i}^{*}(Y)-y_{i}^{k}}
$$

for $i=1, \ldots, d$. Note that $\lambda_{i}^{0 k} \leq \frac{1}{\epsilon_{i}}$. On the other hand, $\lambda_{i}^{0 k} \geq \delta_{i}>0$, where

$$
\delta_{i}=\min _{y \in P(Y)} \frac{1}{B_{i}^{*}(Y)-y_{i}}
$$

Let $\lambda_{\text {max }}^{k}=\max _{i=1, \ldots, d}\left\{\lambda_{i}^{0 k}\right\}$. Then, we normalize the vector $\lambda^{0 k}$ :

$$
\lambda_{i}^{k}=\frac{\lambda_{i}^{0 k}}{\lambda_{\max }^{k}}
$$

Now we have $0<\delta \leq \lambda_{i}^{k} \leq 1$, where $\delta=\min _{i, j}\left\{\delta_{i} \epsilon_{j}\right\}$, for all $k \in\{1, \ldots, K\}$. Thus, in this case the surrogate value function looks as follows

$$
\tilde{v}\left(y, B^{*}(Y), y^{k}\right):=-\max _{i=1, \ldots, d}\left\{\lambda_{i}^{k}\left(B_{i}^{*}(Y)-y_{i}\right)\right\} .
$$

Since $y^{k} \in P(Y)$, it is the solution of (8), where instead of $B(Y)$, the point $B^{*}(Y)$ is used. Moreover, the following equalities hold:

$$
\lambda_{i}^{k}\left(B_{i}^{*}(Y)-y_{i}^{k}\right)=\lambda_{j}^{k}\left(B_{j}^{*}(Y)-y_{j}^{k}\right)
$$

for all pairs $i, j=1, \ldots, d$. Since the value of the Chebyshev function is interpreted as the participant's regret, the equalities (11) mean that the parameters $\lambda_{i}, i=1, \ldots, d$, can be interpreted as the regret coefficients. They balance the regrets associated with losses related to deviations from the bliss point for different criteria. Note that the only normalized vector $\lambda^{k}$ results in $y^{k} \in P(Y)$.

We then propose the following arbitration rule. For each goal point $y^{k}, k=1, \ldots, K$, we consider the vector $\lambda^{k}$ given by (9), (10). We, then, perform the following operations to find the arbitration point:

1. Find the average vector of parameters (vector of average regret coefficients):

$$
\lambda^{a}:=\frac{1}{K} \sum_{k=1}^{K} \lambda^{k} .
$$


2. Find the arbitration decision $x^{a}$ by solving the problem

$$
\min _{x \in X} \max _{i=1, \ldots, d}\left\{\lambda_{i}^{a}\left(B_{i}^{*}(Y)-f_{i}(x)\right)\right\}
$$

In the case of several optimal solutions of the problem, an additional procedure may be needed to find a unique $x^{a}$. Let $y^{a}=f\left(x^{a}\right)$.

As for the case of heuristic arbitration schemes, the solution $x^{a}$ is Pareto optimal, symmetric and anonymous and is independent of irrelevant alternatives in the aforementioned sense.

An illustration of the arbitration rule is given in Appendix.

\section{$5 \quad$ Experiments}

There are, basically, two critical issues concerning the application of the approach described in this paper: one is related to the ability of lay stakeholders to understand the IDM technique and identify their feasible goals. Another issue is the ability of stakeholders to accept an arbitrated decision, however fair it could be. For the experimental study of these issues, the technique was implemented as a web experiment, which was run with different groups in Spain and Russia. We used arbitration based on Chebyshev regret function.

There were three rounds of experiments. At a first round, the technique was tested on a three-criterion problem of a university department budget elaboration problem, see Efremov and Ríos Insua (2007). The participants in the experiment were the department staff. All participants reported that they had managed the task. The average time from entering the experiment until selecting a goal was about a quarter of an hour. Some participants privately communicated their a priori preferences on the problem to the experimenter. For example, one of the participants had a position that could be formulated as follows: "favour investigators and give students what remains". Nevertheless, they could see that their strong wish to follow their principled position, that is, a single criterion optimisation, would bring them to an unreasonable solution, and they changed their preferences when selecting the final goal. This evidence supports the inference that the technique is intelligible in this particular decision-making problem.

Two other rounds of experiments were run on the basis of a five-criterion problem of choosing a hostel in London by a group of friends for a joint trip ${ }^{3}$. We have prepared a paper, see Efremov et al.(2008), with details of the experiments and related issues; here we only sketch the issues relevant to the problem of intelligibility of the multicriteria technique and its role in the decision-making process. In the second round, experiments with non-sophisticated users without mathematical or technical university education (sportsmen, first year students, musicians, etc.) were carried out. In the course of the experiments the participants were proposed to understand the IDM technique without external help. All first year students and musicians managed to understand the IDM technique by themselves, though one third of the students reported difficulties in mastering the technique. However, all sportsmen required support of the instructor. More than one half of the participants (18 participants) assured that they would use the technique in future. It is interesting that all sportsmen and musicians as well as $40 \%$ of students (about $2 / 3$ of participants) used simplified strategies to select their goals, managing only two

\footnotetext{
${ }^{3}$ One can have a look at the web site of the experiment at http://refrenof.escet.urjc.es:8080/rgmas_en/
} 
criteria (price and distance from the city centre) instead of the five given. Nevertheless, the rest of participants seemed to consider all five criteria, thus taking fully into account the multicriteria nature of the problem. In addition, it was found that the participants experienced lack of user-friendliness working with the software.

The third round of experiments was run with senior applied mathematics students. Starting from the assumption that these students can easily master the IDM technique, the third round was devoted mainly to the study of the post-arbitration decision-making procedures. Note that we do not consider the arbitration point we find to be some kind of established truth. We assume that the solution(s) found at the arbitration stage might help to support discussion or voting. The important issue here is that even if some standard mechanism as voting is used to solve a particular participatory decision problem after the procedure we have described in the paper, the participants would vote with a deeper knowledge of the problem. Three different groups participated. The results of the formal arbitration turned out to be very close to the alternative chosen by direct voting by fifth year students who indeed planned to travel to London. The results of arbitration for other (younger) groups were not so close to their final decision since their preferences were changing in time. Note that even though the results of applying formal arbitration to participant's preferences were different from group to group, the final alternative, chosen in an informal deliberation process, was the same in all cases. Thus, one could say, that differences between the groups, which represent the same social environment, are smaller than differences in arbitration results, which are very sensitive to the goals expressed by the participants. This evidence stresses the importance of a thorough deliberation process after applying any formal rule in order to obtain a robust result.

\section{Conclusion}

We have proposed a framework to support participatory decision making through web that does not require too much sophistication from the participants. This is a scenario that will be more and more usual as e-democracy tools are developed. Consequently, techniques for supporting non-sophisticated decision makers are becoming increasingly relevant. The framework goes through two stages: first, the stakeholders apply a simple visual tool, the Interactive Decision Maps technique, which helps them to identify their feasible goals; second, we use an arbitration rule to form the decision. The main original feature of our approach consists of using preference information in the form of feasible goals instead of full description of value functions. In this paper we showed that such limited information can, however, be used in various heuristic and value function-based arbitration schemes. Most classic arbitration rules can be applied if we use the goals to construct surrogate value functions of stakeholders.

There are several issues that we are currently developing. Namely, we are running a systematic experiment on the prototype web-based implementation of our framework. In the experiments with non-sophisticated participants, we have found that most of them are able to master the technique, though they may prefer to use simple self-developed heuristics instead. However, about $20 \%$ of the people without mathematical or technical background failed to understand the technique without being supported by an instructor. This encourages us to refine the methods and software and run additional experiments. We are also developing axiomatic foundations of the arbitration rules we apply. 


\section{Acknowledgment}

The research of R. Efremov was supported by a young post-doctoral foreign researchers grant of the Ministry of Education and Science of Spain (MEC), by the EDEMOCRACIACM project of the University department of Madrid Region, TSI2004-06801-C04-01 project of the Ministry of Education and Science (MEC) of Spain and the TED-ESF program.

The research of D. Ríos Insua was supported by TSI2004-06801-C04-01 MEC project, EDEMOCRACIA-CM, the TED-ESF program and the COST Action IC0602.

The research of A. Lotov was supported by the TED-ESF program, by the grant of the President of Russian Federation for State Support of Leading Scientific Schools (project no. NSh-2982.2008.1), by the Russian Foundation for Basic Research (project no. 07-01-00472) and by a grant of MEC awarded to the PhD program at the Rey Juan Carlos University.

We are very grateful to the referees' comments and discussion.

\section{Appendix. Illustrative examples}

Let us first consider an arbitration procedure based on the application of linear surrogate value functions described in Subsection 4.3.1, i.e.

$$
\tilde{v}(y, w):=\sum_{i=1}^{d} w_{i} y_{i}
$$

where $w=\left(w_{1}, \ldots, w_{d}\right)$ is a vector of positive weights. We assume that $H(Y)$ is polyhedral. The weights can be found by using the idea described in Subsection 4.3.1. Namely, $w^{k}=c^{k}$, where $c^{k}$ is the vector defining the unique supporting hyperplane of $H(Y)$ at $y^{k}$, where $k=1, \ldots, K$. Let us assume that the vectors $c^{k}$ are normalized for all $k=1, \ldots, K$. Since $H(Y)$ is polyhedral and $y^{k} \in P(Y)$, we can assume that $c^{k}>0$ for all $k=1, \ldots, K$.

Let us assume that we use the utilitarian solution, see e.g. Thompson (1994), to construct the arbitration scheme, i.e. we want to maximize

$$
V(y):=\sum_{k=1}^{K} \tilde{v}^{k}\left(y, w^{k}\right)
$$

Then, by using the KKT optimality condition, the vector

$$
\left(\frac{\partial V}{\partial y_{1}}, \ldots, \frac{\partial V}{\partial y_{d}}\right)=\left(\frac{\partial \sum_{k=1}^{K} \tilde{v}^{k}}{\partial y_{1}}, \ldots, \frac{\partial \sum_{k=1}^{K} \tilde{v}^{k}}{\partial y_{d}}\right)=\sum_{k=1}^{K} c^{k}
$$

must belong to the cone of the vectors defining the supporting hyperplanes at the arbitration point $y^{a}$ that maximizes the sum of the individual value functions. Let us denote this vector by $c^{a}$ and consider the linear optimization problem (5) with $w^{a}=c^{a}$. The set of its solutions contains the arbitration point $y^{a}$.

This discussion suggests that the arbitration procedure based on the utilitarian solution could look as follows:

1. Find the support vectors $c^{k}$ for all goal points $y^{k}$ and normalize them.

2. Sum up the support vectors $c^{k}: c^{a}:=\sum_{k=1}^{K} c^{k}$. 
3. Find the arbitration decision $x^{a}$ by solving the following optimization problem $\max _{x \in X} \tilde{v}\left(f(x), c^{a}\right)$, where $\tilde{v}\left(f(x), c^{a}\right)=\sum_{i=1}^{d} c_{i}^{a} f_{i}(x)$.

Should the solution of such optimization problem be not unique, an additional procedure would be needed to define a unique decision $x^{a}$.

Let us consider an example that shows that such arbitration rule has undesirable prop-

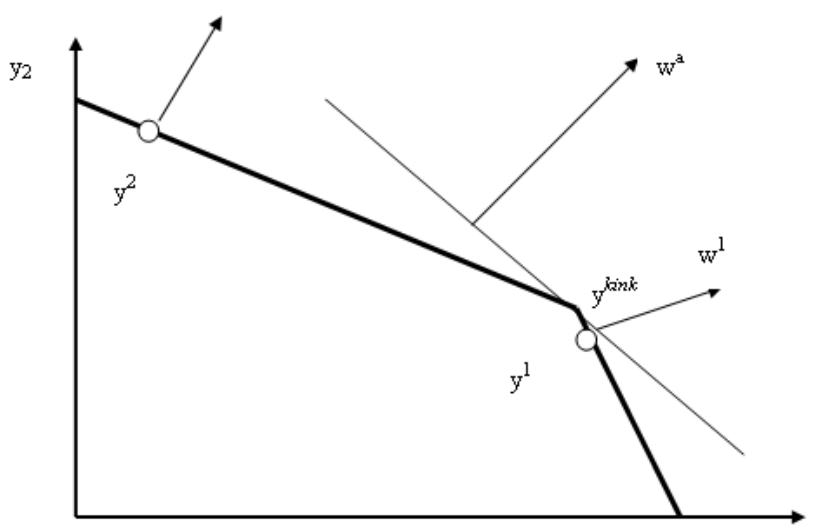

Y1

Figure 4: Illustration of the example with linear weights

erties. Consider first the case of two stakeholders and two criteria, see Figure 4. It is assumed that the first stakeholder has identified the feasible goal $y^{1}$, whereas the second one has identified the goal $y^{2}$. Let the goal $y^{1}$ be located close to the kink point. It is easy to realize that, due to the form of the Pareto frontier, the arbitration point $y^{a}$ is given by the kink point $y^{k i n k}$.

Let us increase now the number of stakeholders. Assume that all of them, except the first one, have identified $y^{2}$ as their feasible goal. However, this will not change the arbitration point $y^{a}$, even if the number of stakeholders tends to infinity. Thus, the participatory decision is defined by the preferences of only one stakeholder.

This example shows that the combination of goal-based surrogate value functions jointly with some arbitration principles may result in an unfair arbitration. Thus, a detailed study of the properties of the arbitration schemes, which use surrogate value function, is needed.

We provide now an illustrative application of the arbitration rule based on the Chebyshev function, by using the same example problem. In Figure 5 one can see the same criterion points $y^{1}$ and $y^{2}$ as in Figure 4. However, the arbitration point for two stakeholders, $y^{a}$, does not coincide with the kink point. One can see now that the arbitration point reflects the stakeholders' preferences: it is located approximately between the individual goals. Let us consider the case of ten stakeholders. Assume, as in the case of the linear surrogate function, that stakeholders with numbers 2 to 10 have identified $y^{2}$. Then, the arbitration point is given by point $y^{10}$, which reflects the preferences of the stakeholders: $y^{10}$ is close to the goal identified by the majority of the stakeholders. Thus, in this particular example, the arbitration rule based on the Chebyshev function is able to somehow take votes into account and provide reasonable arbitration points. 


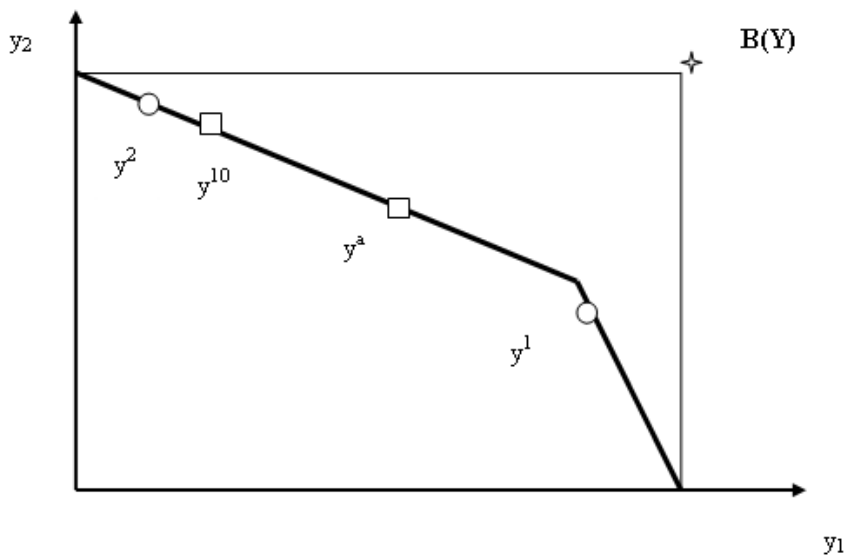

Figure 5: Illustration of the example with Chebyshev weights

\section{References}

Browning, G. (2002). Electronic democracy: Using the Internet to transform American politics (2nd ed.). Medford, NJ: CyberAge Books/Information Today.

Bushenkov, V.A., and Lotov A.V. (1982) Methods for the construction and application of generalized reachable sets: Moscow, Computing Center of the USSR Academy of Sciences (in Russian).

Castelletti A., and Soncini-Sessa R. (2006) A procedural approach to strengthening integration and participation in water resource planning: Environmental Modelling \& Software, Vol. 21, No. 10 pp. 1455 - 1470.

Charnes, A., and Cooper, W. W. (1961) Management Models and Industrial Applications of Linear Programming, vol. 1: New York, John Wiley \& Sons, Inc.

Cohon, J. (1978) Multiobjective Programming and Planning: New York, Academic Press

Dietrich J., Schumann A. H., Lotov A. V. (2006) Workflow oriented participatory decision support for integrated river basin planning: Topics on System Analysis and Integrated Water Resource Management, Edited by A. Castelletti and R. Soncini Sessa, Elsevier, pp. 207-221.

Efremov R., Ríos Insua D. (2007) An experimental study of a web-based framework for group decision support with applications to participatory budget elaboration. International Journal of Technology, Policy and Management, Vol. 7, No.2 pp. 167 $-177$.

Efremov R. , Lotov A. V., Moukhacheva A. (2008) Experimental study of a prototype multiobjective participatory decision aid webtool based on Pareto frontier visualization, Technical Reports on Statistics and Decision Sciences (TR08/08), U. Rey Juan Carlos.

French, S., D. Rios Insua and F. Ruggeri (2007) e-Participation and Decision Analysis, Decision Analysis, Vol. 4, No. 4, pp. 211 - 226. 
Gass, S., and Saaty, T. (1955) The computational algorithm for the parametric objective function: Naval Research Logistics Quarterly, 2, 39.

Germeier, Yu.B. (1970) Introduction to Operations Research: Moscow, Nauka Publishing House (in Russian).

Ignizio, J.P. (1985) Introduction to Linear Goal Programming: Sage Publications, Inc, Beverly Hills.

Keeney, R.L., and Raiffa, H. (1976) Decisions with Multiple Objectives: Preferences and Value Tradeoffs: Wiley, New York.

Kersten G.E., Strecker S.E., and Law K.P. Protocols for Electronic Negotiation Systems: Theoretical Foundations and Design Issues. In K. Bauknecht, M. Bichler, and B. Proll (eds) Lecture Notes in Computer Science, v. 3182, pp. 106-115, New York, Springer.

Larichev, O. (1992) Cognitive validity in design of decision aiding techniques: J. MultiCriteria Decision Analysis, 1(3), 127-138.

Lotov A.V., Bushenkov V., Kamenev G., Loucks D. and Camara A. (1998) Water Resource Conflict Resolution Based on Interactive Tradeoffs Display, Restoration of Degraded Rivers. In: D.P.Loucks (Ed) Challenges, Issues and Experiences, Kluwer Academic Publishers.

Lotov A.V., Bushenkov V.A., and Kamenev G.K. (1999) Feasible Goals Method. Mathematical foundations and ecological applications: Mellen Press, New York, USA (in Russian).

Lotov A.V., Bushenkov V.A., and Kamenev G.K. (2001) Feasible Goals Method. Search for smart decisions: Computing Centre of Russian Academy of Sciences, Moscow.

Lotov A.V. (2003) Internet Tools for Supporting of Lay Stakeholders in the Framework of the Democratic Paradigm of Environmental Decision Making: J. Multi-Criteria Decision Analysis, 12, 145-162.

Lotov A.V., Bushenkov V.A., and Kamenev G.K. (2004a) Interactive Decision Maps. Approximation and Visualization of Pareto Frontier: Kluwer Academic Publishers.

Lotov A., Kistanov A., and Zaitsev A. (2004b) Visualization-based Data Mining Tool and its Web application. In: Y. Shi, W. Xu, and Z. Chen (Eds.), Lecture Notes in Artificial Intelligence, Springer-Verlag Berlin Heidelberg, 3327, XIII, 263.

Lotov A., Kamenev G., Berezkin V., and Miettinen K. (2005) Optimal Control of Cooling Process in Continuous Casting of Steel Using a Visualization-Based Multi-Criteria Approach: Applied Mathematical Modelling, 29(7), 653-672.

McQuaid, M.J., Ong, T.-H., Chen, H., and Nunamaker, J.F. (1999) Multidimensional scaling for group memory visualization. Decision Support Systems, 27, pp. 163176.

Miettinen, K.M. (1999) Nonlinear multiobjective optimization: Kluwer Academic Publishers, Boston.

Podinovski V.V., Nogin, V.D. (1982) Pareto-optimal solutions of multiobjective problems: Nauka, Moscow (in Russian).

Raiffa, H. (1982) The Art and Science of Negotiations: Belknap Press, Harvard University. 
Ríos J., Ríos Insua D. (2007) A framework for participatory budget elaboration support, Journal of the Operational Research Society 59, 268-276.

Ríos, J., Ríos Insua D., and Kim J. B. (2005) Arbitration and Mediation Schemes: Balanced Increment and Balanced Concession: Technical Reports, Rey Juan Carlos University.

Ríos Insua, D., Holgado, J., and Moreno, R. (2004) An e-negotiation system to support e-democracy: J. Multicriteria Decision Analysis, 12, 213-218.

Romero, C. (1991) Handbook of Critical Issues in Goal Programming: Pergamon Press.

Roy, B. (1972) Decisions avec criteres multiples: Metra International, 111, 121-151.

Steuer, R. (1986) Multiple Criteria Optimization, John Wiley, New York.

Wierzbicki, A.P. (1982) A mathematical basis for satisficing decision making: Mathematical Modelling, 3, no. 25, 391-405.

Thomson, W. (1994) Cooperative Models of Bargaining. In: R.J. Aumann and S. Hart (Ed.), Handbook of Game Theory, II, 35, 1238-1284. 